\title{
Dynamics Modeling and Analysis of Spacecraft Antenna Based on Kane Method
}

\author{
Xiaotao Zhou \\ Xi'an Institute of Space Radio Technology, China, 710100 \\ zxtemc_2016@163.com
}

Keywords: Rod antenna structure; Kane method; dynamic stiffening

\begin{abstract}
With the increasing demand and complexity of spacecraft on-orbit tasks, higher requirements are put forward for the dynamic behavior prediction of spacecraft attachment structures. It is difficult to analyze some models effectively by traditional dynamic analysis methods. This paper analyzes the structure of the spacecraft rod antenna and combines the Kane dynamics method, lists the dynamic equations of the Kane form, compares the difference between the Kane method and the traditional modeling method, and draws Kane. The method can calculate and analyze the dynamic stiffening problem. The two models are solved by MATLAB. It is concluded that the dynamic model established by Kane method has more accurate conclusion than the traditional method.
\end{abstract}

\section{Introduction}

During the maneuvering of the spacecraft, the rigid body motion is coupled with the flexible motion generated by the flexible attachment (also known as the rigid-flexible coupling phenomenon). When the spacecraft performs a wide range of motion, the rigid-flexible coupling phenomenon is also called the power. The phenomenon of rigidity. In response to such problems, early studies applied the inertial forces caused by large-scale motion to the deformation motion in the form of loads, not counting the coupling of large-scale motion and structural vibration deformation. Later, the mixed coordinate method was adopted, but it was also limited to the case where the motion range was small and the rate was small. At present, more and more space missions have large flexible attachments, and they are developing in the direction of light weight and high control precision. Therefore, it is necessary to propose a more accurate dynamic model in dynamics research. Kane et al. ${ }^{[1]}$ originally studied the large-scale elastic movement and proposed the phenomenon of " Dynamic stiffening ".

The Kane dynamics method is essentially another expression of the Apell equation. The dynamic equation uses the generalized rate as an independent variable. The generalized velocity is used to derive the partial velocity, and the conventional physical quantities such as displacement and velocity are described, combined with the relevant physical equations. Write general dynamics and general inertial forces, and finally write the dynamic equation ${ }^{[2]}$.

In this paper, the dynamics modeling and analysis of the spacecraft rod antenna is carried out. The beam antenna is equivalent to the beam model for analysis and processing, which can not only reveal the influence of the dynamic stiffness phenomenon in the dynamic modeling process, but also simplify the calculation. The Kane method is used for modeling, and the model is solved and calculated by combining MATLAB. Due to the characteristics of the dynamic equation, the Wilson- $\theta$ method is also used to solve the problem. The simulation results and finite element analysis results show that the kane method can be used to solve the dynamic behavior of the cantilever beam model with dynamic stiffening under the condition of large-scale maneuver.

\section{Kinematics description of the Kane method}

In order to make the dynamic equations of the beam model easier to write, the following assumptions are made for the beam:

1) The beam is Euler-Bernoulli straight beam, the material is uniform and isotropic; 
2) The elastic axis of the section is collinear with the central axis, regardless of the shear deformation and the influence of the moment of inertia of the section;

3) The axial deformation of the beam is small deformation relative to the lateral deformation.

The schematic diagram of the cantilever beam is shown in Figure 1.

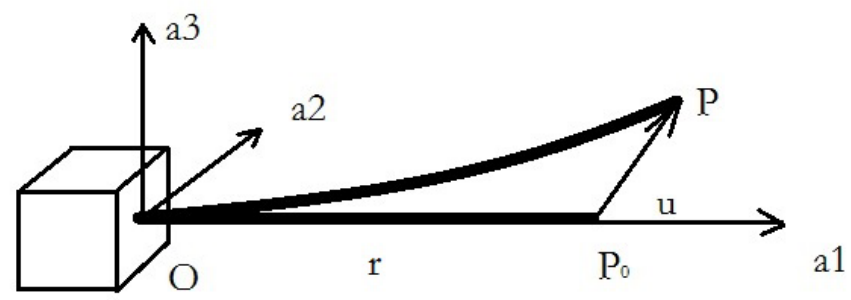

Figure 1 Cantilever beam under wide-range motion conditions

In the figure $\left(a_{1}, a_{2}, a_{3}\right)$ is the unit basis vector of the floating coordinate system on the cantilever beam. $\mathrm{P}$ is the position of the cantilever beam under a wide range of motion conditions. When the cantilever beam is not deformed at rest, $\mathrm{P}$ and $P_{0}$ coincide.

The speed of point $\mathrm{P}$ in the inertial system can be expressed as:

Where: $v_{o}$---- is the velocity vector of the origin $\mathrm{O}$ in the inertial coordinate system

$w_{a}$---- is the angular velocity vector of the floating coordinate system coordinate system in the inertial coordinate system

r---- is the position vector of $P_{0}$ in the conjoined coordinate system

u---- is the deformation vector of $P_{0}$ in the conjoined coordinate system

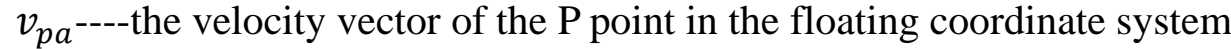

The components of the above formula are:

$$
\begin{gathered}
v_{0}=v_{1} a_{1}+v_{2} a_{2}+v_{3} a_{3} \\
w_{a}=w_{1} a_{1}+w_{2} a_{2}+w_{3} a_{3} \\
v_{p a}=\dot{u}_{1} a_{1}+\dot{u}_{2} a_{2}+\dot{u}_{3} a_{3} \\
r=x a_{1}
\end{gathered}
$$

Substituting the above formula:

$$
\begin{aligned}
& v_{p}=\left[v_{1}+\dot{u}_{1}+w_{2} u_{3}-w_{3} u_{2}\right] a_{1} \\
& +\left[v_{2}+\dot{u}_{2}-w_{1} u_{3}+w_{3}\left(x+u_{1}\right)\right] a_{1} \\
& +\left[v_{3}+\dot{u}_{3}+w_{1} u_{2}-w_{2}\left(x+u_{1}\right)\right] a_{1}
\end{aligned}
$$

Derived from the above formula:

$$
a_{p}=\frac{d v_{p}}{d t}+w_{a} \times v_{p}=C_{1} a_{1}+C_{2} a_{2}+C_{3} a_{3}
$$

In the above formula: 


$$
\begin{aligned}
& C_{1}=\dot{v}_{1}+w_{2} v_{3}-w_{3} v_{2}+\ddot{u}_{1}+\left(\dot{w}_{2}+w_{1} w_{3}\right) u_{3}+2 w_{3} \dot{u}_{3} \\
& -2 w_{3} \dot{u}_{2}+\left(w_{1} w_{2}-\dot{w}_{3}\right) u_{2}-\left(w_{2}^{2}+w_{3}^{2}\right)\left(x+u_{1}\right) \\
& C_{2}=\dot{v}_{2}+w_{3} v_{1}-w_{1} v_{3}+\ddot{u}_{2}+\left(\dot{w}_{3}+w_{1} w_{2}\right)\left(x+u_{1}\right)+2 w_{3} \dot{u}_{1} \\
& -2 w_{1} \dot{u}_{3}+\left(w_{2} w_{3}-\dot{w}_{1}\right) u_{3}-\left(w_{1}^{2}+w_{3}^{2}\right) u_{2} \\
& C_{3}=\dot{v}_{3}+w_{1} v_{2}-w_{2} v_{1}+\ddot{u}_{3}+\left(\dot{w}_{1}+w_{2} w_{3}\right) u_{2}+2 w_{1} \dot{u}_{2} \\
& -2 w_{2} \dot{u}_{1}+\left(w_{1} w_{3}-\dot{w}_{2}\right)\left(x+u_{1}\right)-\left(w_{1}^{2}+w_{2}^{2}\right) u_{3}
\end{aligned}
$$

In order to avoid premature linearization and the result is not accurate enough, for the reasonable description of the deformation field of the elastic body, an arc length variable $s$ and two Cartesian coordinate variables $u_{2} 、 u_{3}$ are used to describe the deformation of the cantilever beam. For the convenience of subsequent solutions, the hypothetical modal method is used to discretize $s, u_{2}, u_{3}$, that is,

$$
\begin{gathered}
s(x, t)=\sum_{j=1}^{\mu_{1}} \phi_{1 j}(x) q_{1 j}(t) \\
u_{2}(x, t)=\sum_{j=1}^{\mu_{2}} \phi_{2 j}(x) q_{2 j}(t) \\
u_{3}(x, t)=\sum_{j=1}^{\mu_{3}} \phi_{3 j}(x) q_{3 j}(t)
\end{gathered}
$$

In the above formula, $\phi_{1 j}(x) 、 \phi_{2 j}(x) 、 \phi_{3 j}(x)$ are hypothetical modal functions, and the natural vibration modes of the $\mathrm{j}$-th order longitudinal, lateral and bending vibrations are obtained when the cantilever beam is slightly vibrated. $q_{1 j}(t) 、 q_{2 j}(t) 、 q_{3 j}(t)$ are the corresponding generalized coordinates, and $\mu_{1} 、 \mu_{2}$ and $\mu_{3}$ are the corresponding modal truncation orders.

In order to adopt the selected generalized coordinates $s, u_{2}, u_{3}$, should be applied to equations (1-5) and (1-6) instead of $u_{1}$.

The selected coordinate description satisfies the following relationship

$$
x+s=\int_{0}^{x}\left[\left(1+\frac{\partial u_{1}}{\partial \sigma}\right)^{2}+\left(\frac{\partial u_{2}}{\partial \sigma}\right)^{2}+\left(\frac{\partial u_{3}}{\partial \sigma}\right)^{2}\right]^{0.5} d \sigma
$$

It can be simplified by two expansions:

$$
s=u_{1}+\frac{1}{2} \int_{0}^{x}\left[\left(\frac{\partial u_{2}}{\partial \sigma}\right)^{2}+\left(\frac{\partial u_{3}}{\partial \sigma}\right)^{2}\right] d \sigma+\ldots
$$

The high order term is omitted in the above equation, so that $s$ is approximated as a linear representation.

Using the above formula to find the partial derivative of $\mathrm{x}$ can be obtained:

$$
\frac{\partial s}{\partial x}=\frac{\partial u_{1}}{\partial x}+\frac{1}{2}\left[\left(\frac{\partial u_{2}}{\partial \sigma}\right)^{2}+\left(\frac{\partial u_{3}}{\partial \sigma}\right)^{2}\right]
$$

The above formula is used when calculating the potential function.

In solving the general inertial force, it is necessary to use the partial velocity, so the partial derivative of $\dot{q}_{r}$ for $v_{p}$ is obtained:

$$
\begin{array}{cc}
\frac{\partial v_{p}}{\partial \dot{q}_{r}}=\phi_{1 j} a_{1} & \left(j=1,2, \ldots, \mu_{1}\right) \\
\frac{\partial v_{p}}{\partial \dot{q}_{r}}=-\sum_{k=1}^{\mu_{2}}\left(\int_{0}^{x} \phi_{2 j, \sigma} \phi_{2 k, \sigma} d \sigma\right) q_{2 k} a_{1}+\phi_{2 j} a_{2} & \left(j=1,2, \ldots, \mu_{2}\right) \\
\frac{\partial v_{p}}{\partial \dot{q}_{r}}=-\sum_{k=1}^{\mu_{3}}\left(\int_{0}^{x} \phi_{3 j, \sigma} \phi_{3 k, \sigma} d \sigma\right) q_{3 k} a_{1}+\phi_{3 j} a_{3} & \left(j=1,2, \ldots, \mu_{3}\right)
\end{array}
$$




\section{Dynamics modeling}

\subsection{Generalized main power}

The ideal cantilever beam strain energy is as follows ${ }^{[3]}$ :

$$
U=\frac{1}{2} \int_{0}^{l} E A\left(\frac{\partial s}{\partial x}\right)^{2} d x+\frac{1}{2} \int_{0}^{l} E I_{3}\left(\frac{\partial^{2} u_{2}}{\partial x^{2}}\right)^{2} d x+\frac{1}{2} \int_{0}^{l} E I_{2}\left(\frac{\partial^{2} u_{3}}{\partial x^{2}}\right)^{2} d x
$$

In turn, the generalized main power can be obtained:

$$
F_{i}=-\frac{\partial U}{\partial q_{r}}
$$

Combined with the basic theory of Kane method:

$$
\begin{gathered}
-F_{1 i}=\sum_{i=1}^{\mu_{1}}\left(\int_{0}^{l} E A \phi_{1 i, x} \phi_{1 k, X} d x\right) q_{1 i} \quad\left(\mathrm{i}=1,2, \ldots, \mu_{1}\right) \\
-F_{2 i}=\sum_{i=1}^{\mu_{2}}\left(\int_{0}^{l} E I_{3} \phi_{2 i, X x} \phi_{2 k, X x} d x\right) q_{2 i} \quad\left(\mathrm{i}=1,2, \ldots, \mu_{2}\right) \\
-F_{3 i}=\sum_{i=1}^{\mu_{3}}\left(\int_{0}^{l} E I_{2} \phi_{3 i, x x} \phi_{3 k, x x} d x\right) q_{3 i} \quad\left(\mathrm{i}=1,2, \ldots, \mu_{3}\right)
\end{gathered}
$$

\subsection{Generalized inertial force}

The generalized inertial force is defined as follows:

$$
F_{i}^{*}=-\int_{0}^{l} \rho \frac{\partial v_{p}}{\partial \dot{q}_{r}} a_{p} d x
$$

The generalized inertial forces corresponding to the generalized coordinates after simplification and calculation are as follows:

$$
\begin{aligned}
& -F_{1 i}^{*}=\left(\dot{v}_{1}+w_{2} v_{3}-w_{3} v_{2}\right) \int_{0}^{l} \rho \phi_{1 i} d x-\left(w_{2}^{2}+w_{3}^{2}\right) \int_{0}^{l} \rho x \phi_{1 i} d x \\
& +\sum_{k=1}^{\mu_{1}}\left[\left(\int_{0}^{l} \rho \phi_{1 i} \phi_{1 k} d x\right) \ddot{q}_{1 k}-\left(w_{2}^{2}+w_{3}^{2}\right) \int_{0}^{l} \rho \phi_{1 i} \phi_{1 k} d x q_{1 k}\right] \\
& -\sum_{k=1}^{\mu_{1}}\left[2 w_{3}\left(\int_{0}^{l} \rho \phi_{1 i} \phi_{2 k} d x\right) \dot{q}_{2 k}-\left(w_{1} w_{2}-\dot{w}_{3}\right) \int_{0}^{l} \rho \phi_{1 i} \phi_{2 k} d x q_{2 k}\right] \\
& +\sum_{k=1}^{\mu_{1}}\left[2 w_{2}\left(\int_{0}^{l} \rho \phi_{1 i} \phi_{3 k} d x\right) \dot{q}_{3 k}+\left(w_{1} w_{3}+\dot{w}_{2}\right) \int_{0}^{l} \rho \phi_{1 i} \phi_{3 k} d x q_{3 k}\right] \\
& \left(i=1,2, \ldots, \mu_{1}\right) \\
& -F_{2 i}^{*}=\left(\dot{v}_{2}+w_{3} v_{1}-w_{1} v_{3}\right) \int_{0}^{l} \rho \phi_{2 i} d x+\left(w_{1} w_{2}+\dot{w}_{3}\right) \int_{0}^{l} \rho x \phi_{2 i} d x \\
& +\sum_{k=1}^{\mu_{2}}\left[\left(\int_{0}^{l} \rho \phi_{2 i} \phi_{2 k} d x\right) \ddot{q}_{2 k}-\left(w_{1}^{2}+w_{3}^{2}\right) \int_{0}^{l} \rho \phi_{2 i} \phi_{2 k} d x q_{2 k}\right] \\
& +\sum_{k=1}^{\mu_{2}}\left[2 w_{3}\left(\int_{0}^{l} \rho \phi_{2 i} \phi_{1 k} d x\right) \dot{q}_{1 k}+\left(w_{1} w_{2}+\dot{w}_{3}\right) \int_{0}^{l} \rho \phi_{2 i} \phi_{1 k} d x q_{1 k}\right] \\
& +\sum_{k=1}^{\mu_{2}}\left[-2 w_{1}\left(\int_{0}^{l} \rho \phi_{2 i} \phi_{3 k} d x\right) \dot{q}_{3 k}+\left(w_{2} w_{3}-\dot{w}_{1}\right) \int_{0}^{l} \rho \phi_{2 i} \phi_{3 k} d x q_{3 k}\right] \\
& -\sum_{k=1}^{\mu_{2}}\left[\left(\dot{v}_{1}+w_{2} v_{3}-w_{3} v_{2}\right)\left(\int_{0}^{l} \rho\left(\int_{0}^{x} \phi_{2 i, \sigma} \phi_{2 k, \sigma} d \sigma\right) d x\right) q_{2 k}\right] \\
& +\sum_{k=1}^{\mu_{2}}\left[\left(w_{2}^{2}+w_{3}^{2}\right)\left(\int_{0}^{l} \rho x\left(\int_{0}^{x} \phi_{2 i, \sigma} \phi_{2 k, \sigma} d \sigma\right) d x\right) q_{2 k}\right] \\
& \left(i=1,2, \ldots, \mu_{2}\right)
\end{aligned}
$$




$$
\begin{aligned}
& -F_{3 i}^{*}=\left(\dot{v}_{3}+w_{1} v_{2}-w_{2} v_{1}\right) \int_{0}^{l} \rho \phi_{3 i} d x+\left(w_{1} w_{3}-\dot{w}_{2}\right) \int_{0}^{l} \rho x \phi_{3 i} d x \\
& +\sum_{k=1}^{\mu_{3}}\left[\left(\int_{0}^{l} \rho \phi_{3 i} \phi_{3 k} d x\right) \ddot{q}_{3 k}-\left(w_{1}^{2}+w_{2}^{2}\right) \int_{0}^{l} \rho \phi_{3 i} \phi_{3 k} d x q_{3 k}\right] \\
& +\sum_{k=1}^{\mu_{3}}\left[-2 w_{2}\left(\int_{0}^{l} \rho \phi_{3 i} \phi_{1 k} d x\right) \dot{q}_{1 k}+\left(w_{1} w_{3}-\dot{w}_{2}\right) \int_{0}^{l} \rho \phi_{3 i} \phi_{1 k} d x q_{1 k}\right] \\
& +\sum_{k=1}^{\mu_{3}}\left[2 w_{1}\left(\int_{0}^{l} \rho \phi_{3 i} \phi_{2 k} d x\right) \dot{q}_{2 k}+\left(w_{2} w_{3}+\dot{w}_{1}\right) \int_{0}^{l} \rho \phi_{3 i} \phi_{2 k} d x q_{2 k}\right] \\
& -\sum_{k=1}^{\mu_{3}}\left[\left(\dot{v}_{1}+w_{2} v_{3}-w_{3} v_{2}\right)\left(\int_{0}^{l} \rho\left(\int_{0}^{x} \phi_{3 i, \sigma} \phi_{3 k, \sigma} d \sigma\right) d x\right) q_{3 k}\right] \\
& +\sum_{k=1}^{\mu_{3}}\left[\left(w_{2}^{2}+w_{3}^{2}\right)\left(\int_{0}^{l} \rho x\left(\int_{0}^{x} \phi_{3 i, \sigma} \phi_{3 k, \sigma} d \sigma\right) d x\right) q_{3 k}\right] \\
& \left(i=1,2, \ldots, \mu_{3}\right)
\end{aligned}
$$

\subsection{Kane dynamic equation}

Substituting the generalized main dynamics and the generalized inertial forces into the Kane dynamic equation can be obtained:

$$
\begin{aligned}
& \sum_{k=1}^{\mu_{1}}\left[M_{i k}^{11} \ddot{q}_{1 k}-\left(w_{2}^{2}+w_{3}^{2}\right) M_{i k}^{11} q_{1 k}+K_{i k}^{1} q_{1 k}\right]-\sum_{k=1}^{\mu_{2}}\left[2 w_{3} M_{i k}^{12} \dot{q}_{2 k}\right. \\
& \left.-\left(w_{1} w_{2}-\dot{w}_{3}\right) M_{i k}^{12} q_{2 k}\right]+\sum_{k=1}^{\mu_{3}}\left[2 w_{2} M_{i k}^{13} \dot{q}_{3 k}+\left(w_{1} w_{3}+\dot{w}_{2}\right) M_{i k}^{13} q_{3 k}\right] \\
& =\left(w_{2}^{2}+w_{3}^{2}\right) X_{1 i}-\left(\dot{v}_{1}+w_{2} v_{3}-w_{3} v_{2}\right) Y_{1 i} \\
& \left(i=1,2, \ldots, \mu_{1}\right) \\
& \sum_{k=1}^{\mu_{2}}\left[M_{i k}^{22} \ddot{q}_{2 k}-\left(w_{1}^{2}+w_{3}^{2}\right) M_{i k}^{22} q_{2 k}+K_{i k}^{2} q_{2 k}\right]+\sum_{k=1}^{\mu_{1}}\left[2 w_{3} M_{i k}^{21} \dot{q}_{1 k}\right. \\
& \left.+\left(w_{1} w_{2}+\dot{w}_{3}\right) M_{i k}^{21} q_{1 k}\right]+\sum_{k=1}^{\mu_{3}}\left[-2 w_{1} M_{i k}^{23} \dot{q}_{3 k}+\left(w_{2} w_{3}-\dot{w}_{1}\right) M_{i k}^{23} q_{3 k}\right] \\
& -\sum_{k=1}^{\mu_{3}}\left[\left(\dot{v}_{1}+w_{2} v_{3}-w_{3} v_{2}\right) K d_{i k}^{2} q_{2 k}\right]+\sum_{k=1}^{\mu_{2}}\left[\left(w_{2}^{2}+w_{3}^{2}\right) K d_{i k}^{2} q_{2 k}\right] \\
& =\left(w_{2}^{2}+w_{3}^{2}\right) X_{2 i}-\left(\dot{v}_{1}+w_{2} v_{3}-w_{3} v_{2}\right) Y_{2 i} \\
& \left(i=1,2, \ldots, \mu_{2}\right) \\
& \sum_{k=1}^{\mu_{3}}\left[M_{i k}^{33} \ddot{q}_{3 k}-\left(w_{1}^{2}+w_{2}^{2}\right) M_{i k}^{33} q_{3 k}+K_{i k}^{3} q_{3 k}\right]+\sum_{k=1}^{\mu_{1}}\left[-2 w_{2} M_{i k}^{31} \dot{q}_{1 k}\right. \\
& \left.+\left(w_{1} w_{3}-\dot{w}_{2}\right) M_{i k}^{31} q_{1 k}\right]+\sum_{k=1}^{\mu_{2}}\left[2 w_{1} M_{i k}^{32} \dot{q}_{2 k}+\left(w_{2} w_{3}-\dot{w}_{1}\right) M_{i k}^{32} q_{2 k}\right] \\
& -\sum_{k=1}^{\mu_{2}}\left[\left(\dot{v}_{1}+w_{2} v_{3}-w_{3} v_{2}\right) K d_{i k}^{3} q_{3 k}\right]+\sum_{k=1}^{\mu_{3}}\left[\left(w_{2}^{2}+w_{3}^{2}\right) K d_{i k}^{3} q_{3 k}\right] \\
& =-\left(w_{1} w_{3}-\dot{w}_{2}\right) X_{3 i}-\left(\dot{v}_{3}+w_{1} v_{2}-w_{2} v_{1}\right) Y_{3 i} \\
& \left(i=1,2, \ldots, \mu_{3}\right)
\end{aligned}
$$

The symbols are defined in the above formula as follows:

$$
\begin{aligned}
& M_{i j}^{l m}=\int_{0}^{l} \rho \phi_{l i} \phi_{m j} d x, X_{l i}=\int_{0}^{l} \rho \phi_{l i} x d x \\
& Y_{l i}=\int_{0}^{l} \rho \phi_{l i} d x, K_{i j}^{1}=\int_{0}^{l} E A \phi_{1 i, x} \phi_{1 j, x} d x \\
& K_{i j}^{2}=\int_{0}^{l} E I_{3} \phi_{2 i, x x} \phi_{2 j, x x} d x, K_{i j}^{3}=\int_{0}^{l} E I_{2} \phi_{3 i, x x} \phi_{3 j, x x} d x \\
& K d_{i j}^{2}=\frac{1}{2} \int_{0}^{l}\left(L^{2}-x^{2}\right) \phi_{2 i, x} \phi_{2 j, x} d x, K d_{i j}^{3}=\frac{1}{2} \int_{0}^{l}\left(L^{2}-x^{2}\right) \phi_{3 i, x} \phi_{3 j, x} d x
\end{aligned}
$$


In the above-mentioned final kinetic equation, the $K d_{i j}^{k}$ term is the dynamic stiffness term. In the derivation of the traditional method, the premature linearization process will lead to the loss of this term.

\section{Simulation analysis}

The parameters of the simulation model are shown in Table 1.

Table 1 Cantilever beam related parameters

\begin{tabular}{c|c|c|c}
\hline Physical properties & Density $/\left(\mathrm{kg} \cdot \mathrm{m}^{-1}\right)$ & Young's modulus $/\left(\mathrm{N} \cdot \mathrm{m}^{-2}\right)$ & Length/m \\
\hline Value & 1.2 & $7 \times 10^{10}$ & 10 \\
\hline Physical properties & $\begin{array}{c}\text { Cross-sectional area } \\
/ \mathrm{m}^{2}\end{array}$ & Moment of inertia $/ \mathrm{m}^{4}$ & Poisson's ratio \\
\hline Value & $4 \times 10^{-4}$ & $2 \times 10^{-7}$ & 0.3
\end{tabular}

In order to reveal the model difference caused by the presence or absence of the dynamic stiffness term, and the coupling of the rigid motion and the elastic motion of the flexible attachment under the large-scale rigid body motion, the Kane dynamic equation obtained from the above formula is revealed. The deformation calculation is performed by using a single-axis fixed-axis rotation. Take the commonly used acceleration rotation equation [3], which has the characteristics of smoothing of the growth and reaching the steady-state angular velocity after the acceleration time $\mathrm{T}$.

$$
\dot{\theta}=\left\{\begin{array}{cl}
\frac{\Omega}{T}\left(t-\frac{T}{2 \pi} \sin \frac{2 \pi t}{T}\right) & 0 \leq t \leq T \\
\Omega & t \geq T
\end{array}\right.
$$

Where $\Omega$ is the steady-state rotational angular velocity and $\mathrm{T}$ is the acceleration time, generally taking $\mathrm{T}=15 \mathrm{~s}$.

The simulation model is rotated in a plane, so $v_{1}=v_{2}=v_{3}=\dot{v}_{1}=\dot{v}_{2}=\dot{v}_{3}=0 ; w_{3}=$ $\dot{\theta}, w_{1}=w_{2}=\dot{w}_{1}=\dot{w}_{2}=0 ; \dot{w}_{3}=\ddot{\theta}$. Substituting these conditions into the kinetic equation can simplify the kinetic equation:

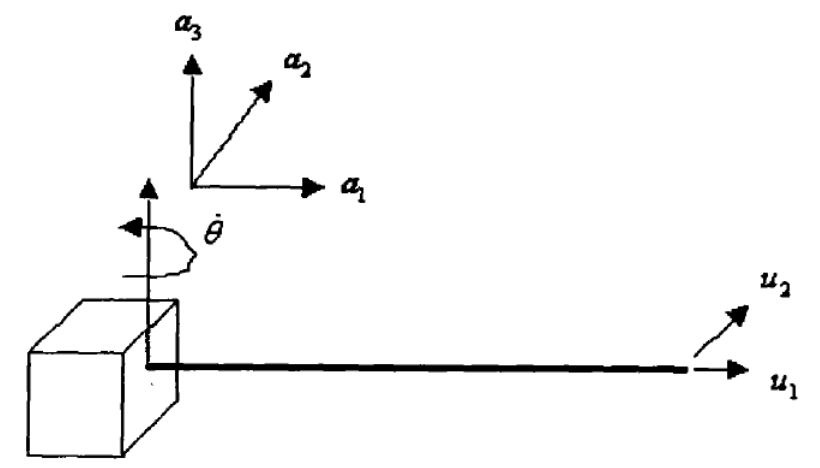

Figure 2 Cantilever beam for fixed axis rotation

$$
\begin{aligned}
& \sum_{k=1}^{\mu_{1}}\left[M_{i k}^{11} \ddot{q}_{1 k}-\dot{\theta}^{2} M_{i k}^{11} q_{1 k}+K_{i k}^{1} q_{1 k}\right]-\sum_{k=1}^{\mu_{2}}\left[2 \dot{\theta} M_{i k}^{12} \dot{q}_{2 k}+\ddot{\theta} M_{i k}^{12} q_{2 k}\right]=\dot{\theta}^{2} X_{1 i} \\
& \left(i=1,2, \ldots, \mu_{1}\right) \\
& \quad \sum_{k=1}^{\mu_{2}}\left[M_{i k}^{22} \ddot{q}_{2 k}-\dot{\theta}^{2} M_{i k}^{22} q_{2 k}+K_{i k}^{2} q_{2 k}\right]-\sum_{k=1}^{\mu_{1}}\left[2 \dot{\theta} M_{i k}^{21} \dot{q}_{1 k}+\ddot{\theta} M_{i k}^{21} q_{1 k}\right] \\
& \quad+\dot{\theta}^{2} \sum_{k=1}^{\mu_{3}} K d_{i k}^{2} q_{2 k}=-\ddot{\theta} X_{2 i}\left(i=1,2, \ldots, \mu_{2}\right)
\end{aligned}
$$

The hypothetical modal function is selected to take the longitudinal free vibration type function of the cantilever beam and the transverse free vibration type function of the cantilever beam, and for the convenience of calculation, only the first order mode is as follows ${ }^{[4]}$ : 


$$
\begin{aligned}
& \phi_{11}(x)=\sin \left(\frac{\pi x}{2 l}\right) \\
& \phi_{21}(x)=\frac{1}{2}\left[\operatorname{ch}\left(\frac{\beta x}{l}\right)-\cos \left(\frac{\beta x}{l}\right)+\frac{\sin \beta-\operatorname{sh} \beta}{\cos \beta+\operatorname{ch} \beta}\left(\operatorname{sh}\left(\frac{\beta x}{l}\right)-\sin \left(\frac{\beta x}{l}\right)\right)\right]
\end{aligned}
$$

In the above formula, $\beta$ is determined by $\cosh \beta \cos \beta+1=0$.

Calculate the modal integral and get the kinetic equation as:

$$
\begin{aligned}
& {\left[\begin{array}{ll}
1 & 0 \\
0 & 1
\end{array}\right]\left[\begin{array}{l}
\eta \\
\ddot{\zeta}
\end{array}\right]+\left[\begin{array}{cc}
0 & -1.3557 \dot{\theta} \\
2.7115 \dot{\theta} & 0
\end{array}\right]\left[\begin{array}{l}
\dot{\eta} \\
\dot{\zeta}
\end{array}\right]} \\
& +\left[\begin{array}{cc}
2.9742 \times 10^{6}-\dot{\theta}^{2} & 0.6779 \dot{\theta} \\
1.3557 \dot{\theta} & 1.1035+0.2674 \dot{\theta}^{2}
\end{array}\right]\left[\begin{array}{l}
\eta \\
\zeta
\end{array}\right]=\left[\begin{array}{c}
9,2351 \dot{\theta}^{2} \\
-12.8512 \theta
\end{array}\right]
\end{aligned}
$$

Where $\eta$ and $\zeta$ are the longitudinal and transverse deformation mode coordinates of the cantilever beam. The above formula is a typical set of rigid differential equations. According to the literature, the Wilson- $\theta$ algorithm in the form of coefficient freeze is used to solve the problem. That is, in the $\Delta \mathrm{t}$ time, the coefficient of the equation is considered to be constant, and the Wilson- $\theta$ algorithm is used to calculate the coordinates in each time period.

According to the reference, the calculation formula of the vibration angular frequency of the transverse vibration of the beam can be obtained, and the first-order vibration angular frequency of the beam is $w_{1}=1.0505 \mathrm{rad} / \mathrm{s}$. The stable rotational speed used in the simulation is less than $w_{1}$, equal to $w_{1}$ and greater than The case of $w_{1}$ is calculated to compare the vibration trend of the beam under the influence of the dynamic stiffness term. Take $w=0.2 w_{1}, w=0.5 w_{1}, w=0.8 w_{1}$, $w=1.0 w_{1}$, under the four conditions, the Kane method which can take the dynamic stiffness term can be compared with the results obtained by the traditional method. Curves are as follow:

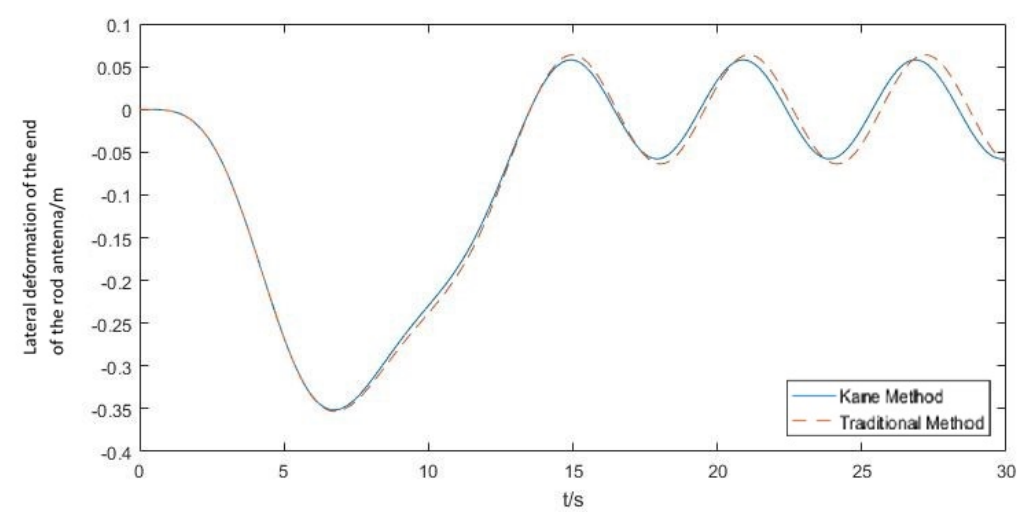

Figure 3 Model comparison curve when $\Omega=0.2 w_{1}$

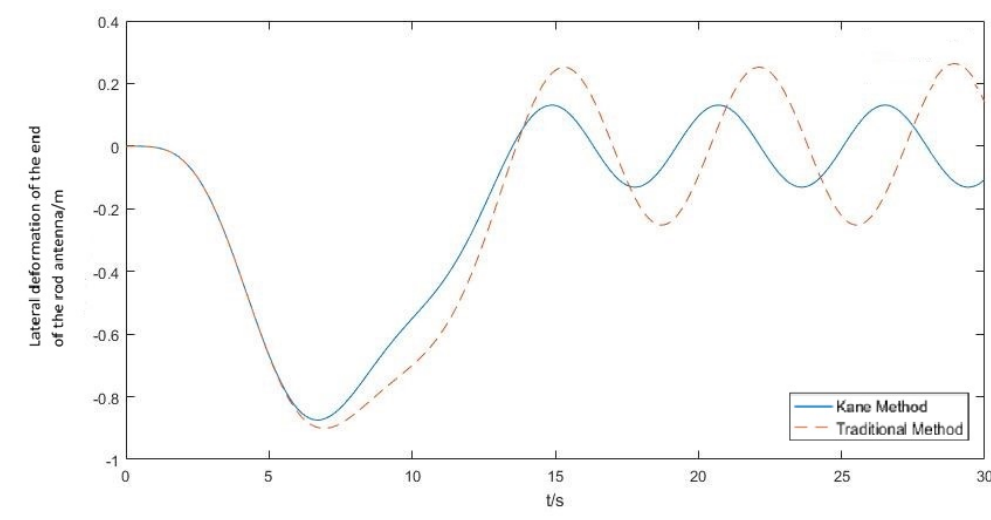

Figure 4 Model comparison curve when $\Omega=0.5 w_{1}$ 


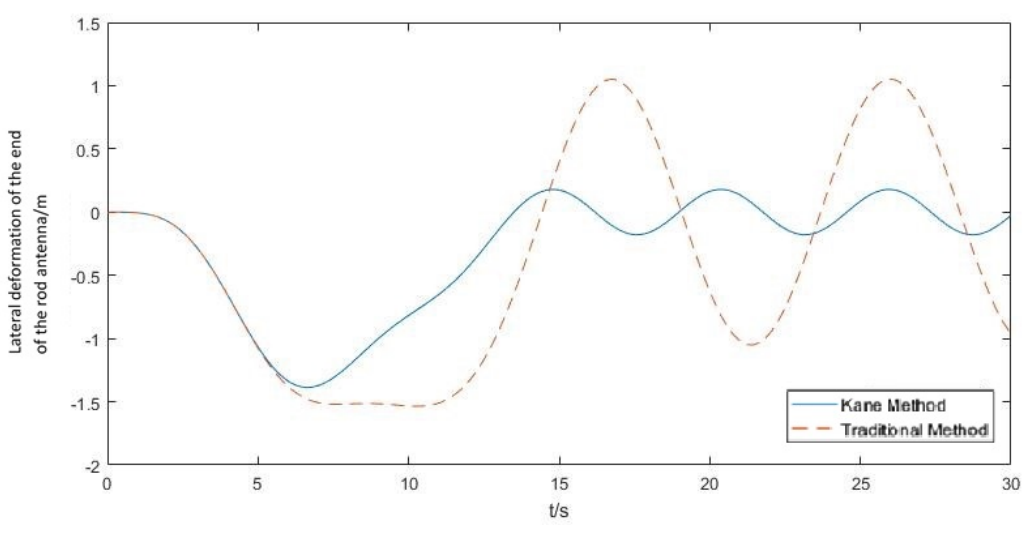

Figure 5 Model comparison curve when $\Omega=0.8 w_{1}$

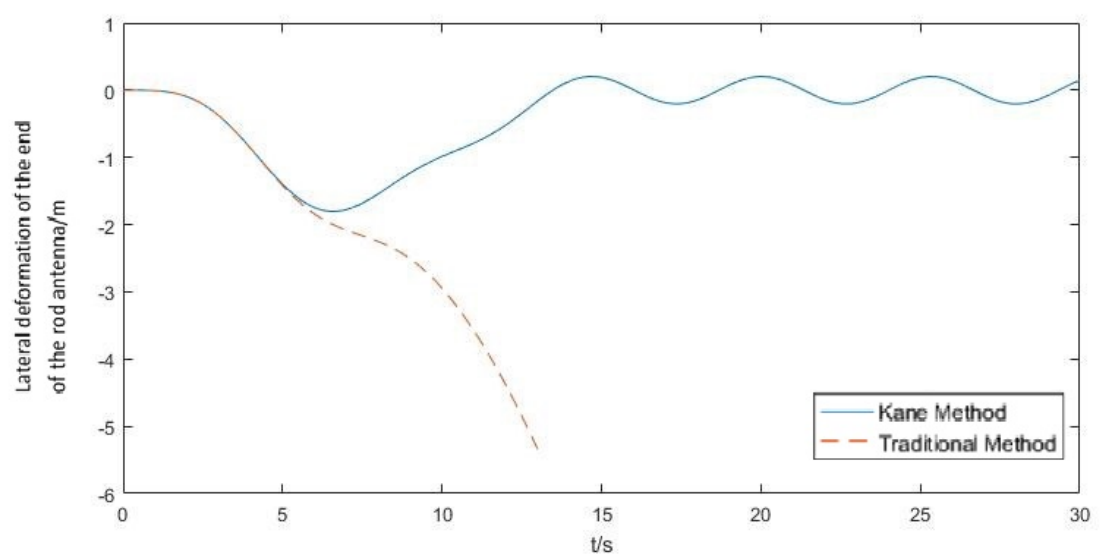

Figure 6 Model comparison curve when $\Omega=w_{1}$

It can be seen from the above simulation curve that when the rotational speed of the central rigid body is not high, the trend of deformation displacement obtained by the traditional zero-order approximate dynamic model and the first approximate dynamic model derived based on the Kane method is not much different, as shown in Fig. 3. However, as the rotational speed of the central rigid body increases, the deviation of the deformation displacement obtained by the conventional method and the motion tendency based on the Kane method become larger and larger, as shown in Fig. 5 and Fig. 6. In the case where the first-order natural frequency is taken as the rotational speed, the simulation results of the conventional method that does not take into account the dynamic stiffness term appear divergent, which is not in accordance with the actual situation.

The Kane method has a steady trend at all rotational angular velocities, and the correctness of the results can be verified from the subsequent finite element analysis on the one hand. On the other hand, the reference to the existing references is the same. the result of.

This phenomenon is the "dynamic stiffening" phenomenon proposed by Kane in the initial analysis. In the traditional zero-order dynamic model, the missing stiffness dynamic stiffness term causes its variation with time, when the stiffness term is reduced to Below 0, the solution obtained by the equation is a divergent solution.

\section{Conclusion}

The simulation analysis of the spacecraft rod antenna for the cantilever beam model shows that under the dynamic model based on the Kane method, the dynamic equation containing the dynamic stiffening term can be obtained. Based on the MATLAB programming simulation analysis results, for the case of any large-scale motion in space, the results are more stable and conform to the physical reality. The traditional model that ignores the dynamic stiffness term in the dynamic equation can obtain relatively stable results when performing small-angle slow motion. However, 
on the basis of a wide range of rapid movements, the results obtained tend to diverge.

The dynamics modeling analysis of the Kane method is still relatively simple for the analysis of the cantilever beam model, but reflects the advantages of the method. Unlike the traditional dynamic modeling method, the obtained dynamic model has a dynamic stiffness term, so that the solution result can be applied to large and fast spacecraft maneuvering processes.

\section{References}

[1] Jiang Jianping. Research on rigid-flexible coupling dynamics of large flexible multi-body structure satellite [D]. Graduate School of National University of Defense Technology, National University of Defense Technology, 2004.

[2] Liu Yanzhu. Higher Dynamics [M]. Higher Education Press, 2016:253-287.

[3] Yoo H H, Ryan R R, Scott R A. Dynamics of flexible beams undergoing overall motions[J]. Journal of Sound and vibration, 1995, 181(2): 261-278.

[4] Zou Jingxiang, Yu Kaiping. Structural Dynamics [M]. Harbin Institute of Technology Press, 2009:90-99.

[5] Huang Wenhu, Cao Dengqing, Han Zengqi. Research Progress and Prospect of Spacecraft Dynamics and Control[J]. Advances in Mechanics, 2012, 42(4): 367-394.

[6] Zhang Yiyao. Research on rigid-flexible coupled dynamics modeling and dynamic characteristics of flexible spacecraft [D]. Harbin Institute of Technology, 2015.

[7] Li Cuichun. Research on rigid-flexible coupled dynamics modeling and attitude control technology for flexible spacecraft [D]. Beijing Institute of Technology, 2014.

[8] Luo Wen. Rigid-flexible coupled dynamics modeling of solar wing satellites [D]. Harbin Institute of Technology, 2015.

[9] Wallrapp O. Linearized Flexible Multibody Dynamics Including Geometric Stiffening Effects*[J]. Journal of Structural Mechanics, 1991, 19(3): 385-409.

[10]Likins P W. Finite element appendage equations for hybrid coordinate dynamic analysis[J]. International Journal of Solids and Structures, 1972, 8(5): 709-731.

[11]Wang J T, Huston R L. Kane's equations with undetermined multipliers-application to constrained multibody systems[J]. Journal of applied mechanics, 1987, 54(2): 424-429.

[12]Huston R L. Dynamics of constrained multibody systems[J]. Journal of Applied Mechanics, 1984, 51: 899.

[13] Singh R P, VanderVoort R J, Likins P W. Dynamics of flexible bodies in tree topology-a computer-oriented approach[J]. Journal of Guidance, Control, and Dynamics, 1985, 8(5): 584-590.

[14]Yoo H H, CHUNG J. Dynamics of rectangular plates undergoing prescribed overall motion[J]. Journal of Sound and Vibration, 2001, 239(1): 123-137.

[15]Kane T R, Ryan R, Banerjee A K. Dynamics of a cantilever beam attached to a moving base[J]. Journal of Guidance, Control, and Dynamics, 1987, 10(2): 139-151.

[16]Kane, T.R. Spacecraft Dynamics [M]. Science Press, 1988. 